\title{
A particle batch smoother for soil moisture estimation using soil temperature observations
}

\author{
Jianzhi Dong, Susan C. Steele-Dunne, Nick van de Giesen \\ Department of Water Management, Faculty of Civil Engineering and Geosciences, Delft University of Technology, \\ Delft, Netherlands.
}

\begin{abstract}
In this study, we present a Particle Batch Smoother (PBS) to determine soil moisture profiles by assimilating soil temperatures at two depths (4 and $8 \mathrm{~cm})$. The PBS can be considered as an extension of the standard particle filter (PF) in which soil moisture is updated within a window of fixed length using all observed soil temperatures in that window. This approach was developed with a view to assimilating temperature observations from distributed temperature sensing (DTS) observations, a technique which can provide temperature observations every meter or less along cables up to kilometers in length. Here, the PBS approach is tested using soil moisture and temperature, and meteorological data from an experimental site in Citra, Florida. Results demonstrate that the PBS provides a statistically significant improvement in estimated soil moisture compared to the PF, with only a marginal increase in computational expense $(<3 \%$ of CPU time). This confirms that assimilating a sequence of temperature observations yields a better soil moisture estimate compared to sequential assimilation of individual temperature observations. The impact of observation interval was investigated for both PF and PBS, and the optimal window length was determined for the PBS. While increasing the observation interval is essential to maintain the spread of particle values in the PF, the PBS performance is best when all available observations are assimilated.
\end{abstract}

Keywords: soil moisture, distributed temperature sensing, data assimilation, particle approaches, smoothing

\section{Introduction}

In recent years, several novel measurement techniques have been developed to provide insight into soil moisture scaling between point scale and satellite footprint scale [1]. These techniques include cosmic-ray soil moisture probe [e.g. 2, 3, 4], GPS reflectometry [e.g. 5, 6] and distributed temperature sensing (DTS) [e.g. 7, 8, 9].

This study is motivated by the potential use of passive DTS to monitor soil moisture. DTS is an advanced temperature measurement technique using fiber optic cables. Using DTS, temperature observations with an accuracy of $0.1{ }^{\circ} \mathrm{C}$, temporal resolution of 1 minute and spatial resolution of 1 meter can be easily achieved along kilometers of cables [10, 11]. Dong et al. [12] demonstrated that soil temperature can be used to directly update soil moisture estimates in a data assimilation system. The prior estimated soil moisture and temperature profiles from a fully coupled soil vapor, heat and water transport model were updated by sequentially assimilating 
temperature observations at surface layers using an Ensemble Kalman Filter (EnKF) [13]. The EnKF assumes the model-prior to be Gaussian distribution, and only uses the first two moments of the distribution (i.e. the mean and the variance). As a result, the EnKF can yield spurious estimates, particularly when the Gaussian assumption is violated [14]. Particle approaches are considered here because they approximate the entire model posterior distribution using Monte Carlo sampling. Thus, instead of preserving just the first two moments of the distribution, they also track the higher moments $[15,16]$. Particle filters have been used to estimate soil moisture and for model parameter estimation [e.g. 17, 18, 19]. They have also been successfully applied in streamflow estimation [e.g. 16, 20, 21].

Particle filtering is not without limitations. One potential disadvantage is that it often requires more particles to obtain the same accuracy as an EnKF with fewer ensemble members [21]. Early applications in hydrology illustrated that resampling could improve the performances of the PF [16]. Resampling the particles after each update involves discarding the particles with negligible weights to avoid particle degeneration. More recently, the Markov Chain Monte Carlo (MCMC) algorithm has increasingly been included in particle filtering to improve the performance of the PF with a limited number of particles [15, 20, 22]. It explores the space around the particles and accepts/rejects new proposed particles using the Metropolis algorithm. This MCMC technique essentially increases the variability of the particle values and allows for better exploration of the state/parameter space. Another alternative is to provide a better proposition density during the weight updating, i.e. to sample more intensively at the locations with larger likelihoods. The proposal distribution is usually calculated using some variant of the Kalman filter [15, 23]. This algorithm is particularly suitable for the cases when the particles with larger likelihoods are at the tails of the prior distribution and is therefore particularly suited to soil moisture estimation [15]. Here, we will consider the particle filter as outlined by Moradkhani et al. [16].

In addition to considering particle approaches rather than ensemble approaches, this study will investigate the potential benefit of using smoothing rather than filtering for this problem. The hypothesis is that series of temperature observations at different depths can be used to monitor the propagation of a thermal wave from the surface into the soil. This propagation is controlled by the thermal properties of the soil, temporal variations in which are dominated by soil moisture at a given location. Hence, assimilating a series of temperature observations should give us more information on soil moisture than sequential assimilation of instantaneous temperature measurements. This is a similar rationale to the application of variational methods (a type of smoothing) to assimilate series of surface soil temperatures to estimate surface energy fluxes [e.g. 24, 25, 26].

In this study, we will present the derivation of the particle smoother algorithm (PBS) and show how it relates to the PF. A particle batch smoother has recently been applied to assimilate remotely-sensed fractional snow covered area data into a land surface model to estimate the snow water equivalent [27]. Here, it will be used to estimate soil moisture by assimilating soil temperature profile observations. In the PBS all temperature observations within a certain window are assimilated in a batch to estimate the evolution of soil moisture within this window.

We will first demonstrate that sequential data assimilation based on particle approaches (PF) can be used to determine moisture by assimilating temperature observations. Then, the optimal observation interval in the PF will be investigated. Next, we will demonstrate that batch smoothing (PBS) outperforms the sequential assimilation (PF) when the same temperature information was used. The optimal assimilation strategy (e.g. observation interval and window length) will be determined for the PBS, and this "optimal" PBS will be compared with the "optimal" PF. Finally, the computation cost of the two algorithms will be compared. Data from in-situ point sensors were used to determine the optimal window length and observation interval for the PBS, 
and to demonstrate that it outperforms the standard PF.

\section{Method and materials}

\section{2.1. Hydrus-1D model}

In this study, the vertical soil water, heat and vapor transport processes in the unsaturated 64 zone are simulated using the Hydrus-1D model [28]. One-dimensional liquid and vapor flow are 65 given by:

$$
\frac{\partial \theta}{\partial t}=\frac{\partial}{\partial z}\left[K_{T h} \frac{\partial h_{p}}{\partial z}+K_{L h}+K_{T T} \frac{\partial T}{\partial z}\right]-S
$$

where $\theta$ is soil water content $\left(\mathrm{m}^{3} \mathrm{~m}^{-3}\right)$ at time $t(s), z$ is the vertical coordinate (positive upward) $(m), h_{p}$ is soil water pressure head $(m)$, and $T$ is soil temperature $(K) . K_{T h}$ and $K_{T T}$ are the isothermal and thermal total hydraulic conductivities, respectively, and $K_{L h}$ is the isothermal unsaturated hydraulic conductivity. $S$ is a sink term $\left(m^{3} m^{-3} s^{-1}\right) . K_{L h}$ and the soil retention curve are determined using van Genuchten's model [29] :

$$
\begin{aligned}
& K_{L h}=K_{s} S_{e}^{l}\left[1-\left(1-S_{e}^{\frac{1}{m}}\right)^{m}\right]^{2} \\
& \theta(h)=\left\{\begin{array}{cc}
\theta_{r}+\frac{\theta_{s}-\theta_{r}}{\left[1+|\alpha h|^{n}\right]^{m}} & h<0 \\
\theta_{s} & h \geq 0
\end{array}\right.
\end{aligned}
$$

where $K_{s}$ is the saturated hydraulic conductivity $\left(m s^{-1}\right), S_{e}$ is the effective saturation, $l, m, n$ and $\alpha$ are empirical shape parameters and $\theta_{r}$ and $\theta_{s}$ are the residual and saturated soil water contents $\left(\mathrm{m}^{3} \mathrm{~m}^{-3}\right)$. The parameters in equation (2) and (3) were estimated using the ROSETTA model [30] using soil texture and bulk density $\left(\rho_{b}\right)$ reported in Section 2.3 .

The governing equation for soil heat transport is expressed as:

$$
\begin{aligned}
\frac{\partial C_{p} T}{\partial t}+L_{0} \frac{\partial \theta_{v}}{\partial t}= & \\
& \frac{\partial}{\partial z}\left[\lambda(\theta) \frac{\partial T}{\partial z}\right]-C_{w} \frac{\partial q_{L} T}{\partial z} \\
& -L_{0} \frac{\partial q_{v}}{\partial z}-C_{v} \frac{\partial q_{v} T}{\partial z}-C_{w} S T
\end{aligned}
$$

where $C_{w}, C_{v}$ and $C_{p}$ are the volumetric heat capacities of water, vapor and moist soil $\left(\mathrm{Jm}^{-3} \mathrm{~K}^{-1}\right)$, $L_{0}$ is the volumetric latent heat of vaporization of liquid water $\left(\mathrm{Jm}^{-3}\right), q_{L}$ and $q_{v}$ are the flux densities of liquid water and vapor $\left(\mathrm{ms}^{-1}\right)$, and $\lambda(\theta)$ is apparent soil thermal conductivity $\left(\mathrm{Wm}^{-1} \mathrm{~K}^{-1}\right)$. $\lambda(\theta)$ is estimated from:

$$
\lambda(\theta)=\lambda_{0}(\theta)+\beta C_{w}\left|q_{L}\right|
$$

where $\beta$ is the thermal dispersivity $(m)$. The Hydrus-1D model is modified to model soil thermal conductivity $\left(\lambda_{0}\right)$ using the model of $\mathrm{Lu}$ [31]. The required parameters are soil texture and bulk density. 
The "Atmospheric boundary condition with surface runoff" is applied to the upper boundary, and "Free drainage" is applied for the lower boundary condition in the soil water model (Equation (1)). The soil surface energy balance is used as the upper boundary for the heat transfer, and "zero gradient" is assumed at the lower boundary. The details of the model and boundary descriptions are provided by [32]. In this study, the model time step is approximately 1 minute, and the soil column to $1 \mathrm{~m}$ depth is simulated with a vertical resolution of $1 \mathrm{~cm}$.

\subsection{Sequential Data assimilation}

The model states of interest are soil moisture and temperature from the surface $(0 \mathrm{~cm})$ to 1 meter. An ensemble of model states is evolved in parallel using the forward model:

$$
\mathbf{x}_{t}^{i}=f\left(\mathbf{x}_{t-1}^{i}, \mathbf{u}_{t}^{i}, \mathbf{b}^{i}\right)+\mathbf{w}_{t}^{i}
$$

where $\mathbf{x}_{t}^{i}$ is the model state ( $\theta$ and $T$ in this study) of the $i^{t h}$ particle at time $t, \mathbf{u}_{t}^{i}$ are the perturbed forcing data, $\mathbf{b}^{i}$ is a vector of time invariant model parameters, $\mathbf{w}_{t}^{i}$ is the model error, and $f$ is the forward model (Hydrus-1D) [20]. The model error $\mathbf{w}_{t}^{i}$ is assumed to be normally distributed with a standard deviation of $0.005 \mathrm{~m}^{3} / \mathrm{m}^{3}$ for soil moisture, and $0.01 \mathrm{~K}$ for soil temperature.

The model estimates are related to the observations by:

$$
\widehat{\mathbf{y}}_{t}^{i}=h\left(\mathbf{x}_{t}^{i}\right)+\mathbf{v}_{t}^{i}
$$

where $\widehat{\mathbf{y}}_{t}^{i}$ is the simulated observation vector, $h$ is a nonlinear operator relating the prior estimated states $\left(\mathbf{x}_{t}^{i}\right)$ to the measured variable and $\mathbf{v}_{t}^{i}$ is the observation error [20]. The observation error is set to be $0.1 \mathrm{~K}$, according to the field calibration of the temperature sensors. In this study, the observations are the temperatures at 4 and $8 \mathrm{~cm}$.

\subsubsection{Particle Filter}

In the particle filter, the posterior distribution of the model state can be written in recursive form as follows [22]:

$$
p\left(\mathbf{x}_{1: t}^{i} \mid \mathbf{y}_{1: t}\right)=p\left(\mathbf{x}_{1: t-1}^{i} \mid \mathbf{y}_{1: t-1}\right) \frac{p\left(\mathbf{x}_{t}^{i} \mid \mathbf{x}_{1: t-1}^{i}\right) p\left(\mathbf{y}_{t} \mid \mathbf{x}_{t}^{i}\right)}{p\left(\mathbf{y}_{t} \mid \mathbf{y}_{1: t-1}\right)}
$$

103 Integrating out $\mathbf{x}_{1: t-1}^{i}$, gives the marginal distribution $[22,33]$ :

$$
p\left(\mathbf{x}_{t}^{i} \mid \mathbf{y}_{1: t}\right)=\frac{p\left(\mathbf{y}_{t} \mid \mathbf{x}_{t}^{i}\right) p\left(\mathbf{x}_{t}^{i} \mid \mathbf{y}_{1: t-1}\right)}{p\left(\mathbf{y}_{t} \mid \mathbf{y}_{1: t-1}\right)}
$$

where $\mathbf{y}_{1: t}$ is the observation vector. This equation is called the updating step in which the likelihood function, $p\left(\mathbf{y}_{t} \mid \mathbf{x}_{t}^{i}\right)$, is used to update the prior estimates. The prior estimates at time step $t$ are described as [33]:

$$
p\left(\mathbf{x}_{t}^{i} \mid \mathbf{y}_{1: t-1}\right)=\int p\left(\mathbf{x}_{t}^{i} \mid \mathbf{x}_{t-1}^{i}\right) p\left(\mathbf{x}_{t-1}^{i} \mid \mathbf{y}_{1: t-1}\right) d \mathbf{x}_{t-1}^{i}
$$

In the particle filter, the model state posterior density is approximated as:

$$
p\left(\mathbf{x}_{1: t} \mid \mathbf{y}_{1: t}\right)=\sum_{i=1}^{N} w_{t}^{i} \delta\left(\mathbf{x}_{1: t}-\mathbf{x}_{1: t}^{i}\right)
$$


where $\delta()$ is the Direc delta function [34]. Because the posterior density is difficult to sample directly, importance sampling is often used to draw particles from a known function $q\left(\mathbf{x}_{1: t} \mid \mathbf{y}_{1: t}\right)$ and assign the weights according to:

$$
w_{t}^{i *}=\frac{p\left(\mathbf{x}_{1: t}^{i} \mid \mathbf{y}_{1: t}\right)}{q\left(\mathbf{x}_{1: t}^{i} \mid \mathbf{y}_{1: t}\right)}
$$

where $w_{t}^{i *}$ is the importance weight. Equation (12) can be expressed as [16]:

$$
w_{t}^{i *} \propto w_{t-1}^{i *} \frac{p\left(\mathbf{y}_{t} \mid \mathbf{x}_{t}^{i}\right) p\left(\mathbf{x}_{t}^{i} \mid \mathbf{x}_{t-1}^{i}\right)}{q\left(\mathbf{x}_{t}^{i} \mid \mathbf{x}_{t-1}^{i}, \mathbf{y}_{t}\right)}
$$

where $p\left(\mathbf{y}_{\mathbf{t}} \mid \mathbf{x}_{t}^{i}\right)$ is the likelihood, $p\left(\mathbf{x}_{t}^{i} \mid \mathbf{x}_{t-1}^{i}\right)$ is the transition prior, i.e. the probability of moving to $\mathbf{x}_{t}^{i}$ from $\mathbf{x}_{t-1}^{i}$, and $q\left(\mathbf{x}_{k+1}^{i} \mid \mathbf{x}_{k}^{i}, \mathbf{y}_{t}\right)$ is the proposal distribution in the importance sampling. Usually, the transition prior is used for the proposal distribution [16]. Hence, Equation (13) can be simplified as:

$$
w_{t}^{i *} \propto w_{t-1}^{i *} p\left(\mathbf{y}_{t} \mid \mathbf{x}_{t}^{i}\right)
$$

The likelihood function is expressed as:

$$
p\left(\mathbf{y}_{\mathbf{t}} \mid \mathbf{x}_{t}^{i}\right)=\frac{1}{(2 \pi)^{n / 2} \operatorname{det}(\mathbf{R})^{1 / 2}} e^{\left[-0.5\left(\mathbf{y}_{t}-\widehat{\mathbf{y}}_{t}\right)^{T} \mathbf{R}^{-1}\left(\mathbf{y}_{t}-\widehat{\mathbf{y}}_{t}^{i}\right)\right]}
$$

where $\mathbf{R}$ is the error covariance of observations, and $n$ is the number of the observations, e.g. $n=2$ in this case (temperatures at 4 and $8 \mathrm{~cm}$ were assimilated). The normalized weight $\left(w_{t}^{i}\right)$ is calculated as:

$$
w_{t}^{i}=\frac{w_{t}^{i *}}{\sum_{i=1}^{N} w_{t}^{i *}}
$$

Fig.1 (top panel) illustrates the PF algorithm at an update step. Initially, the uniformly distributed weights are assigned to the particles. When observations are available, the PF will update the weights of each particle according to the prior distribution and likelihood (i.e. Equation (15)). As shown in Fig.1(a), the PF adds weights to the particles closer to the observation. Resampling is usually required to prevent weight degeneration, i.e. a situation where most of the particles have negligible weights. Moradkhni et al. [16] demonstrated that resampling the posterior after each update will avoid the degeneracy problem, and result in a significantly improved performance. A detailed description of resampling is given by Moradkhni et al. [16]. Therefore, we also perform resampling when the particle weights are updated (Fig.1b), which results in updated states (Fig.1c).

\subsubsection{Particle Batch Smoother}

Fig.1 (lower panel) illustrates the implementation of the PBS. While the PF assimilates observations sequentially, the PBS assimilates all of the observations within a window in a single batch. Therefore, the posterior density is calculated for a series of model states in a window $(L)$ i.e. $p\left(\mathbf{x}_{t-L+1: t} \mid \mathbf{y}_{1: t}\right)$.

Similar to the PF algorithm, this marginal distribution is used to derive the weights updating equation:

$$
w_{t, s}^{i *} \propto w_{t-L, s}^{i *} p\left(\mathbf{y}_{t-L+1: t} \mid \mathbf{x}_{t-L+1: t}^{i}\right)
$$




$$
w_{t, s}^{i}=\frac{w_{t, s}^{i *}}{\sum_{i=1}^{N} w_{t, s}^{i *}}
$$

where the subscript $s$ denotes smoother. The calculated weights will be assigned to the entire window, i.e. $w_{t-L+1: t, s}^{i}=w_{t, s}^{i}$. The likelihood function is calculated based on the observations of the entire window:

$$
p\left(\mathbf{y}_{t-L+1: t} \mid \mathbf{x}_{t-L+1: t}^{i}\right)=\prod_{j=t-L+1}^{t} \frac{1}{(2 \pi)^{n / 2} \operatorname{det}(\mathbf{R})^{1 / 2}} e^{\left[-0.5\left(\mathbf{y}_{j}-\overline{\mathbf{y}}_{j}^{i}\right)^{T} \mathbf{R}^{-1}\left(\mathbf{y}_{j}-\widehat{\mathbf{y}}_{j}^{i}\right)\right]}
$$

Clearly, the PF algorithm can be considered as a special case of the PBS algorithm where $L=1$. The update procedure is the same as illustrated in Fig.1, except that the state vector includes all states within that window. After resampling, the algorithm will move to the next window with all the weights initialized to $1 / N$. Different observations intervals can be used in the PBS algorithm, as shown in Fig .1 (d) and (e). When a longer observation interval is used, the PBS will discard the observations collected between the observation intervals.

\subsection{Experimental Data}

Data from the Microwex-2 experiment will be used to test and compare the performance of the PF and PBS. This experiment was conducted from 17 March to 3 June 2004, by the Center for Remote Sensing, Agricultural and Biological Engineering Department, at the Plant Science Research and Education Unit of the university of Florida, Gainesville. A full description of this experiment is given by Judge et al. [35]. Soil temperature and moisture data were collected at 5 depths $(2,4,8,32$ and $64 \mathrm{~cm})$, from 26 March. Meteorological data (e.g. precipitation, air temperature, relative humidity and wind speed) were collected every 15 minutes. The sand, silt and clay content of $89.5 \%, 3.4 \%$ and $7.1 \%$ mean the soil at the site is classed as sand in the USDA soil textural classification. The corn was planted on 18 March. Here we focus on the period with negligible biomass (26 March to 10 April).

\subsection{Data assimilation set-up}

The distribution assumed for each perturbed model input is shown in Table 1.We are interested in investigating whether the proposed algorithms can handle the biases/uncertainties in the soil properties, when only rough soil texture classification information is available. This is often the case in distributed temperature sensing applications where soil texture can vary considerably over the scale of the installation [7]. Sand and silt content were sampled uniformly within the USDA classes of sand and sandy loam, i.e. sand from $55 \%$ to $95 \%$ and silt from $0 \%$ to $20 \%$. This is to represent the spatial variability of the soil texture. In a typical DTS installation, the soil class or USDA soil texture classification might be known in general, but not the exact soil texture at each meter of cable. Multiplicative errors distributions were used for the forcing data to avoid obtaining negative values for precipitation, radiation, relative humidity or wind speed. The standard deviation of the errors applied to the forcing represent the instrument errors, and the spatial variability. Forcing data and the soil temperature data were collected at different locations. The initial soil moisture and temperature profile were assumed to be vertically uniform. The initial values were sampled from a uniform distribution $\left[0.05 \mathrm{~m}^{3} \mathrm{~m}^{-3}, 0.25 \mathrm{~m}^{3} \mathrm{~m}^{-3}\right]$ for soil moisture, and $\left[17^{\circ} \mathrm{C}, 23^{\circ} \mathrm{C}\right]$ for soil temperature. A previous synthetic study has shown that temperature observations at two depths in the shallow subsurface provide sufficient information to estimate soil 
moisture using the EnKF [12]. In this study, soil temperature temperature observations at 4 and $8 \mathrm{~cm}$ were used to update the full soil temperature and moisture profiles.

The objectives of this study are to demonstrate that particle-based data assimilation can determine soil moisture by assimilating soil temperature observations in real world data, and that particle batch smoothing yields better results than filtering.

First, we will demonstrate that the particle filter can be used to assimilate soil temperature observations at two depths into the Hydrus-1D model to estimate soil moisture estimates. In this first test, an observation interval of 45 minutes will be assumed, and the number of the particles will be set to be 100. Results from the PF will be compared to a model open loop (OL) run, which is identical to running the 100 particles without performing any assimilations. Results from the PF and OL are also compared to in-situ observations of soil moisture throughout the profile.

Second, we will determine the optimal observation interval in the PF algorithm. With DTS, observations can be made every minute. However, assimilating these observations too frequently can limit the range of particle values. If this becomes too small, the observation may fall outside the particle range, resulting in a poor update. To examine the impact of increasing observation intervals on the range of particle values, we will vary this interval from 15 minutes to 3 hours. Due to the limited duration of the experiment, the initial condition has a significant influence on the estimated soil moisture, particularly at depth. To account for this, experiments are repeated 20 times with different initial conditions, and the median and range of RMSE will be used to determined the optimal observation interval for the PF.

Next, we will focus on the PBS algorithm. We will examine the impact of observation interval on the PBS algorithm. As in the PF, the observation interval may affect the performance. The observation interval will be varied from 15 minute to 1.5 hour. In this case, a window length of 3 hours will be assumed. Then we will investigate the impact of assumed window length on the PBS estimate. As shown in Section 2.2, if the window length (L) is one (i.e. the window length is equal to the observation interval), the PBS and the PF are equivalent. Our hypothesis is that the evolution of temperature in time contains more information on soil moisture than the relationship between instantaneous soil moisture and temperature. Hence, increasing the window length should lead to an improved estimate. However, increasing the length of the window increases the dimension of the distributions to be estimated. This may increase the number of particles required, and hence the computational expense. Maintaining the observation interval of 15 minutes, the window length will be varied from 1 to 12 hours. Then we will compare the optimal particle filter and the optimal particle batch smoother.

Finally, we consider the number of particles required by the two optimal approaches. Though we expect the PBS to yield an improved estimate, this approach may require more particles and therefore greater computational expense. A trade-off is necessary between the reduction in RMSE and the increase in computational demand between the two approaches. The PF and the PBS will be compared as the number of particles is increased from 10 to 300 .

\section{Deterministic and probabilistic performance assessment}

The RMSE is calculated as following:

$$
R M S E=\sqrt{\frac{1}{N_{t}} \sum_{t=1}^{N_{t}}\left(\bar{\theta}_{t}-\theta_{t, o b s}\right)^{2}}
$$


where $N_{t}$ is the number of total time steps that has observations, $\bar{\theta}_{t}$ is the mean of the particle estimates at time step $t$, and $\theta_{t, o b s}$ is the observed soil moisture at $t$. The absolute bias of the estimates will also be calculated as:

$$
\text { Bias }=\left|\frac{1}{N_{t}} \sum_{t=1}^{N_{t}}\left(\bar{\theta}_{t}-\theta_{t, o b s}\right)\right|
$$

Probabilistic verification tools Quantile - Quantile (Q-Q) plot and reliability $\left(\alpha_{r}\right)$ will be used for assessing the performance of the proposed algorithms. Probabilistic metrics are mainly concerning whether the uncertainty of the estimates (particle range or ensemble spread) is appropriate, instead of the accuracy of the estimates. The quantile of the predictive distribution is calculated at each time step [36]:

$$
z_{t}=\frac{1}{N} \sum_{i=1}^{N} k_{i}
$$

where $z_{t}$ is the quantile of the predictive distribution calculated at time $t, k_{i}=1$ when $\theta_{t, o b s}>\theta_{t, i}$, and $k_{i}=0$, otherwise [37]. In the perfect case, the cumulative distribution of $z_{t}$ should be the same as the cumulative uniform distribution $(U[0,1])$. If $z_{t}$ clustered at the middle range, it indicates the uncertainty is overestimated. The uncertainty is underestimated when the $z_{t}$ clustered around the tails. In the case that $z_{t}$ is constantly lower/higher than $U[0,1]$, it indicates the estimates are biased [38]. Based on the Q-Q plot, the reliability $\left(\alpha_{r}\right)$ of the estimates can be computed as:

$$
\alpha_{r}=1-\frac{2}{N_{t}} \sum_{t=1}^{N t}\left|z_{t}-U[0,1]\right|
$$

The reliability $\left(\alpha_{r}\right)$ varies from 0 (zero reliability) to 1 (perfect reliability).

\section{Results and discussion}

\subsection{The particle filter}

Fig. 2 shows an example of the soil moisture profile estimated using the particle filter $(\mathrm{PF})$ when temperature observations at $4 \mathrm{~cm}$ and $8 \mathrm{~cm}$ are assimilated every 45 minutes. Note that the case with a significantly biased initial soil moisture condition is presented here. In the DTS implementation, the soil moisture is supposed to be measured every meter up to kilometers by assimilating soil temperatures at shallow soil depths. Hence, it is impossible to provide correct initial soil moisture profiles everywhere along the DTS installation. As a result, we are interested in the ability of the proposed data assimilation approach to correct for errors in the initial condition. The first thing to note is that the estimated soil moisture from the PF is generally closer to the observed soil moisture than that from the open loop (OL). The OL generally overestimates soil moisture at all depths. The range of particle values in the OL (indicated in pink) is due to the sources of uncertainty described in Table 1. Because the OL estimate is never constrained by observations, the impact of uncertainty in the initial condition persists through the study interval. The range of soil moisture values from the OL generally even exceeds the dynamic range of the observed soil moisture.

The greatest improvements due to the PF are observed at $4 \mathrm{~cm}$ and $8 \mathrm{~cm}$, which are the depths at which soil temperatures were assimilated. At $4 \mathrm{~cm}$, the RMSE is reduced from 0.069 to $0.039 \mathrm{~m}^{3} \mathrm{~m}^{-3}$ and at $8 \mathrm{~cm}$ it is reduced from 0.074 to $0.045 \mathrm{~m}^{3} \mathrm{~m}^{-3}$. The soil moisture estimates 
are improved when the PF resamples the particles with larger likelihoods. The soil moisture estimates at $2 \mathrm{~cm}$ are also greatly improved (from 0.060 to $0.037 \mathrm{~m}^{3} \mathrm{~m}^{-3}$ ), primarily by resampling the particles that provide larger likelihoods at 4 and $8 \mathrm{~cm}$. This resampling also has a significant impact on the range of particles. It generally takes about a day for the PF to shed the influence of the uncertain initial condition. Due to uncertainty in precipitation, the range in soil moisture between 2 and $8 \mathrm{~cm}$ from the PF increases after precipitation. Assimilation with the PF has limited impact at $32 \mathrm{~cm}$ and $64 \mathrm{~cm}$ (Fig.2 (d) and (e)). Because of the sandy soil and high evaporative demand, there is little variability in soil moisture in response to precipitation at these depths. The estimated soil moisture is largely determined by the prescribed initial condition. The lack of correlation between soil moisture and temperature at this depth means that assimilation is ineffective. The only manner in which soil moisture at these depths is corrected is through the eventual impact of the updated surface $(2$ to $8 \mathrm{~cm}$ ) soil moisture on the lower layers through the model physics. The lower soil moisture values in the PF compared to the OL between $2 \mathrm{~cm}$ and $8 \mathrm{~cm}$ eventually lead to drier soil at $32 \mathrm{~cm}$ (after April 1) and $64 \mathrm{~cm}$ (after April 3). Fig.3 gives some additional insight into how and when the PF is most effective in updating soil moisture. Fig.3 (a) and (b) show the influence of uncertainty in shortwave radiation and soil texture on the soil temperatures at 4 and $8 \mathrm{~cm}$. The spread of particle values is largest during sunlight hours and at a maximum in the afternoon. Fig.3 (c) shows the spread of particle values of soil moisture after a precipitation event. Note that the PF is ineffective until just before noon when the spread of temperature values is large enough for the PF to update temperature and hence soil moisture. This is also apparent at $8 \mathrm{~cm}$ (Fig.3 (d)). This explains why the soil moisture estimated in the PF is not updated immediately after precipitation in Fig.2.

Fig.4 shows the impact of observation interval on the performance of the PF. From Fig.2, it is clear that the initial condition can influence the estimate, particularly at depth. Therefore, results for this experiment are presented in terms of box plots constructed using 20 cases, each of which has a different initial condition. Hence, Fig. 4 also provides insight into the robustness of the PF to diffrent initial conditions. From 2 to $8 \mathrm{~cm}$ (Fig.4 (a) to (c)), the median RMSE from the PF is always lower than that of the OL. At greater depth (Fig.4 d and e), the median RMSE is close to that of the OL regardless of the observation interval. The range of RMSE values is also larger than that closer to the surface. Recall that the particle range of soil moisture at depths reduces dramatically after a few updates. The limited influence of precipitation and temperature at depth mean that the particle range at depths will not grow again. Therefore, if the initial distribution of particles leads to the PF updating soil moisture towards an incorrect value, it is difficult to correct the estimates towards the observations. At $32 \mathrm{~cm}$ and $64 \mathrm{~cm}$, Fig. 4 shows that the estimated soil moisture can even be worse than that from the OL.

With less frequent assimilation, the median PF RMSE approaches that of the OL. Assimilating more often than every 45 minutes yields little improvement in terms of median RMSE, but the interquartile range (IQR) and full range of values is often higher at lower observation intervals. The particle filter can only adjust the weights of particles, so the range of particle values must be wide enough to include the true value if the estimate is to be correct. Due to the observation error is very small in this study, assimilating too frequently prevents the range of values from growing and can therefore yield a poorer estimate. Though no distinct RMSE minimum is shown in Fig.4 between assimilation interval of 30 to 120 minutes, the IQR seems to be the minimum when assimilation interval is 45 minutes. For the purpose of comparison, 45 minutes is considered to be the optimal observation interval for the PF, which will be compared with the PBS estimates. 


\subsection{The Particle Batch Smoother}

First, we will compare the PF and the PBS algorithms assuming they both use an observation interval of 45 minutes (PF-45min and PBS-45min in Table 2). The window length for the PBS is 3 hours, i.e. the PBS will update temperature and soil moisture every 45 minutes in a 3 hour window using all temperature observations (at $4 \mathrm{~cm}$ and $8 \mathrm{~cm}$ ) within that window. Table 2 shows the RMSE of soil moisture estimated using the PF and the PBS. The PBS results in a statistically significant reduction in RMSE compared to the PF (using a two-tailed paired T-test, $p<0.05$ ). This is analogous to the improvement observed by using an ensemble batch smoother compared to an ensemble Kalman filter (e.g. [39]). Furthermore, by assimilating a series of temperature observations, the PBS exploits the influence of soil moisture in propagating a thermal wave from the surface into the soil [26]. This is a stronger relationship than that between instantaneous temperature and soil moisture values.

The advantage of using the PBS algorithm is also shown in the reduction of the bias, and the increased reliability. At depth above $32 \mathrm{~cm}$, the reliability of the PBS is nearly twice as high as that in the PF.

Despite resampling, the PF reduces the range of particle values every time there is an update as it proceeds sequentially through the 3 hour period. The PBS, on the other hand, allows the range of particle values to grow over the 3 hour window. Across all model time steps in the whole study period, the range of the particle values is on average $17 \%$ larger than that of the $\mathrm{PF}$ algorithm. Consequently, the observations are more likely to fall within the range of values considered by the PBS.

\subsubsection{Impact of Observation interval}

Fig. 5 shows the influence of the observation interval on the PBS algorithm. The total number of the observations within the assumed 3 hour window is varied from 2 (1.5 hour interval) to 12 (15 minute assimilation interval). If the observation interval is equal to or greater than 45 minutes, the PBS yields little if any improvement over the PF with an observation interval of 45 minutes (shown in black dashed line for reference). However, unlike the PF algorithm (Fig .4), the median RMSE consistently increases with increased assimilation interval. This is particularly noticeable between $2 \mathrm{~cm}$ to $8 \mathrm{~cm}$. Therefore, the best results are obtained when all available observations are assimilated. This may prove particularly useful in the context of DTS as observations can be made every minute or less.

\subsubsection{Impact of Window length}

The impact of window length in the PBS algorithm is explored in Fig.6, where an observation interval of 15 minutes, and 100 particles are used. From $2 \mathrm{~cm}$ to $32 \mathrm{~cm}$, increasing the window length from 1 to 3 hours results in a reduction in the median RMSE. The improvement is greatest at the assimilation depths of $4 \mathrm{~cm}$ and $8 \mathrm{~cm}$. This confirms that assimilating a series of temperature observations may contain more soil moisture information than sequentially assimilation of instantaneous observations. However, increasing the window length further to 6 or 12 hours leads to an increase in both median RMSE and the IQR. There are two contributors to this degradation in performance. First, when precipitation occurs within a window, the estimated soil moisture prior to the precipitation event may be drawn to a moister condition by the subsequent observations. This was observed when brightness temperature was assimilated to estimate soil moisture using an ensemble batch smoother [39]. Second, the dimension of the distributions to be estimated increases with window length, and additional particles may be needed to accurately capture the posterior distribution. 


\subsubsection{Comparison of the "optimal" $P F$ and the PBS approaches}

Based on the results in Fig.5 and Fig .6, the optimal assimilation strategy for the PBS combines a 15 minutes observation interval with a window length of 3 hours. Fig. 7 shows an example of soil moisture estimated using this optimal PBS and the optimal PF (45 minute observation interval). The initial particle settings are the same for both algorithms. The soil moisture at all depths (Fig.7) benefit from the significant improvement in the correction from the assumed initial conditions. The PBS draws the estimate to the truth within the first day, while the PF adjusts it slightly before the particle range collapses inhibiting further improvement. At $32 \mathrm{~cm}$ and $64 \mathrm{~cm}$, the PBS estimate continues to track the truth as the impact of improved soil moisture at $2 \mathrm{~cm}$ to $8 \mathrm{~cm}$ is propagated downwards through the model physics; while the PF estimate is drying out at $8 \mathrm{~cm}$ to $64 \mathrm{~cm}$, the PBS increases at a similar rate to the true observed soil moisture. At $2 \mathrm{~cm}$ and $4 \mathrm{~cm}$, the PBS also draws the moisture estimates closer towards observations after precipitation (e.g. 30 Mar, 01, 03 and 07 Apr). The Q-Q plot in Fig .8 compares the PF and the PBS from a probabilistic point of view. In general, the PF estimates are biased compared to the observed soil moisture, since the predicted quantiles are consistently lower than the uniform distribution. As discussed above, this is caused by the errors in the initial condition, and the uncertainties in the parameters. Compared with the PF, the PBS reduces the bias of the estimates, which is most significant at depth of $32 \mathrm{~cm}$. Both the PF and the PBS are overconfident in the estimates, i.e. the particle range are too narrow to encompass the observations. This is partly because soil moisture was not directly assimilated, and partly because the observation accuracy of soil temperature is very small, which leads to particle weight degeneracy.

Results in Fig.7 and Fig.8 are illustrative and based on one assumed initial condition. Table 2 shows the averaged RMSE from 20 tests, each with a different assumed initial condition. It is clear that the optimal PBS (PBS-15min) yields a significant reduction in RMSE compared to the optimal PF (PF-45min). Similar to the results in Fig.7, the greatest improvements are at $32 \mathrm{~cm}$ and $64 \mathrm{~cm}$ where the RMSE is reduced by 0.023 and $0.020 \mathrm{~m}^{3} \mathrm{~m}^{-3}$ respectively. The bias in the PBS-15min is significantly smaller than that of PF- $45 \mathrm{~min}$ estimated soil moisture. This may indicates that the PBS algorithm is more suitable in correcting the errors in the initial conditions. The reliability of the PBS-15min is approximately 4 to 7 times higher than that of PF-15min at depths above $8 \mathrm{~cm}$. At 32 and $64 \mathrm{~cm}$, the differences in reliability are insignificant. This indicates that both algorithm are overconfident in the estimates, which is consistent with Fig.8. The PBS may be further improved by including a MCMC algorithm [e.g. 20, 22], or by modifying the likelihood function to a distribution with heavy tails [40]. Both techniques may help to identify the location with larger posterior probability, and reduce the overconfidence of the estimates.

\subsection{Computational burden of the $P B S$ and the $P F$}

Here, we quantify the potential increase in computational burden associated with using a smoothing approach (PBS) rather than a filter (PF). There are two factors to consider. The first is that the posterior distribution has a larger dimension in the PBS as so additional particles may be required to capture it. Fig.9 (a) to (e) show the impact of the number of particles on the RMSE in soil moisture at each depth. For any given number of the particles, the PBS outperforms the $\mathrm{PF}$ at each depth. For both algorithms, the median and the range of the RMSE is generally lower for a larger number of particles. The greatest reduction in RMSE is observed between $2 \mathrm{~cm}$ and $8 \mathrm{~cm}$ as the number of particles is increased up to 100. Any further increase leads to a marginal, if any, reduction in median RMSE for both algorithms.

The second is the additional cost of performing the sequential importance sampling calculations for the larger state vector. Furthermore, the states at previous times are stored in memory 
which adds input/output costs. Fig.9 (f) shows that the difference in clock time required by the PBS and PF is negligible $(<3 \%)$. The dominant control is the number of particles, so the computational burden is determined by the Hydrus-1D simulations rather than the PF or PBS update steps.

\section{Conclusion}

In this study, we investigated the potential to use particle approaches to estimate soil moisture from temperature observations. Two particle-based approaches (i.e. the particle filter, PF and the particle batch smoother, PBS) were tested by assimilating temperature observations and validating the estimated soil moisture profile against soil moisture observed using conventional Hydra probes. The PBS uses the evolution of soil temperature within a window, instead of instantaneous measurements. Therefore, the PBS may be more suitable for capturing the temperature heating/cooling rate, hence more suitable for soil moisture estimation. We considered sources of uncertainty comparable to those which would be encountered in a DTS application, i.e uncertain meteorological forcing, soil texture parameters etc.

Results demonstrate that assimilation using a particle filter yields a significant improvement over an open loop (no assimilation) run. The best estimates were obtained between the surface and the depth of the deepest temperature observation. Estimates at greater depth were particularly sensitive to the prescribed initial condition as the range of particle values collapsed soon after the first updates preventing any additional update. The particle filter updates the states by updating the weights of the particles, giving those that agree with the observations a larger weight. It can only adjust within the range of predicted values, so it is essential that this range includes the observation. Results indicate that the PF performs best when observations are assimilated at an interval that allows this predicted range to grow. Further research will investigate whether including MCMC and/or alternative proposal distributions could alleviate this problem.

In addition, we implemented a particle batch smoother algorithm, in which a series of temperature observations within a window are assimilated to update the trajectory of soil moisture in that window. Results demonstrated that this smoothing approach yielded a statistically significant reduction in RMSE compared to the particle filter. Furthermore, this improvement was achieved with a negligible increase in computational cost. The PBS uses a trajectory of temperature observations within a window, which contains more moisture information than one instantaneous observation. This results in reduction of RMSE up to $0.023 \mathrm{~m}^{3} \mathrm{~m}^{-3}$, compared with PF algorithm. The PBS updates the prior moisture estimates once per window, which allows us to use all available observations without particle degeneration. As the PBS performs assimilation on the entire window at once, the dimension of the joint distribution of the states is larger than in the PF. For soil moisture estimates, a window length of 3 hour with observation frequency of 15 minutes was shown to yield the best results. For both the PF and PBS, the number of particles was found to be about 100. Increasing the number any further resulted in only a marginal improvement in RMSE, while the computational burden increased linearly with the number of particles.

The approaches studied here were developed to use Distributed Temperature Sensing to estimate soil moisture. Applying the particle approaches with DTS would allow us to estimate soil moisture every $25 \mathrm{~cm}$ to $1 \mathrm{~m}$ along fiber-optic cables that can be several kilometers in length. Though these first results are already promising, ongoing research will consider the additional benefit of performing dual state-parameter estimation, e.g. [16, 41]. Improving the soil parameters, particularly the soil thermal properties, would improve the performance of the model itself and potentially provide a means to monitor soil heat flux using DTS. 


\section{Acknowledgement}

The author thank Dr. Jasmeet Judge at University of Florida providing model forcing and soil property data. The first author was financially supported for his Ph.D. research by the China Scholarship Council with the project reference number of 201206040043. The work of S. C. Steele-Dunne was supported by The Netherlands Organisation for Scientic Research (NWO) Veni Grant Program (ALW 863.09.015).

\section{Reference}

[1] T. E. Ochsner, M. H. Cosh, R. H. Cuenca, W. A. Dorigo, C. S. Draper, Y. Hagimoto, Y. H. Kerr, E. G. Njoku, E. E. Small, and M. Zreda, "State of the art in large-scale soil moisture monitoring," Soil Science Society of America Journal, vol. 77, no. 6, pp. 1888-1919, 2013.

[2] M. Zreda, D. Desilets, T. Ferré, and R. L. Scott, "Measuring soil moisture content non-invasively at intermediate spatial scale using cosmic-ray neutrons," Geophysical research letters, vol. 35, no. 21, 2008.

[3] T. E. Franz, M. Zreda, T. Ferre, R. Rosolem, C. Zweck, S. Stillman, X. Zeng, and W. Shuttleworth, "Measurement depth of the cosmic ray soil moisture probe affected by hydrogen from various sources," Water Resources Research, vol. 48 , no. $8,2012$.

[4] J. Dong, T. E. Ochsner, M. Zreda, M. H. Cosh, and C. B. Zou, "Calibration and validation of the cosmos rover for surface soil moisture measurement," Vadose Zone Journal, vol. 13, no. 4, 2014.

[5] K. M. Larson, E. E. Small, E. D. Gutmann, A. L. Bilich, J. J. Braun, and V. U. Zavorotny, "Use of gps receivers as a soil moisture network for water cycle studies," Geophysical Research Letters, vol. 35, no. 24, 2008.

[6] C. C. Chew, E. E. Small, K. M. Larson, and V. U. Zavorotny, "Effects of near-surface soil moisture on gps snr data: development of a retrieval algorithm for soil moisture," Geoscience and Remote Sensing, IEEE Transactions on, vol. 52, no. 1, pp. 537-543, 2014.

[7] S. C. Steele-Dunne, M. M. Rutten, D. M. Krzeminska, M. Hausner, S. W. Tyler, J. Selker, T. a. Bogaard, and N. C. van de Giesen, "Feasibility of soil moisture estimation using passive distributed temperature sensing," Water Resources Research, vol. 46, p. W03534, Mar. 2010.

[8] C. Sayde, C. Gregory, M. Gil-Rodriguez, N. Tufillaro, S. Tyler, N. van de Giesen, M. English, R. Cuenca, and J. S. Selker, "Feasibility of soil moisture monitoring with heated fiber optics," Water Resources Research, vol. 46, p. W06201, June 2010 .

[9] F. Ciocca, I. Lunati, N. Van de Giesen, and M. B. Parlange, "Heated optical fiber for distributed soil-moisture measurements: A lysimeter experiment," Vadose Zone Journal, vol. 11, no. 4, 2012.

[10] S. W. Tyler, J. S. Selker, M. B. Hausner, C. E. Hatch, T. Torgersen, C. E. Thodal, and S. G. Schladow, "Environmental temperature sensing using raman spectra dts fiber-optic methods," Water Resources Research, vol. 45, no. 4, 2009.

[11] J. S. Selker, L. Thévenaz, H. Huwald, A. Mallet, W. Luxemburg, N. van De Giesen, M. Stejskal, J. Zeman, M. Westhoff, and M. B. Parlange, "Distributed fiber-optic temperature sensing for hydrologic systems," Water Resources Research, vol. 42, no. 12, p. W12202, 2006.

[12] J. Dong, S. C. Steele-Dunne, T. E. Ochsner, and N. van de Giesen, "Determining soil moisture by assimilating soil temperature measurements using the ensemble kalman filter," Submitted to Advances in Water Resources.

[13] G. Evensen, "Sequential data assimilation with a nonlinear quasi-geostrophic model using monte carlo methods to forecast error statistics," Journal of Geophysical Research: Oceans (1978-2012), vol. 99, no. C5, pp. 10143-10162, 1994.

14] C. M. DeChant and H. Moradkhani, "Examining the effectiveness and robustness of sequential data assimilation methods for quantification of uncertainty in hydrologic forecasting," Water Resources Research, vol. 48, no. 4, 2012.

[15] H. Bi, J. Ma, and F. Wang, "An improved particle filter algorithm based on ensemble kalman filter and markov chain monte carlo method," IEEE Journal of selected topics in applied earth observations and remote sensing.

[16] H. Moradkhani, K.-L. Hsu, H. Gupta, and S. Sorooshian, "Uncertainty assessment of hydrologic model states and parameters: Sequential data assimilation using the particle filter," Water Resources Research, vol. 41, no. 5, 2005.

[17] C. Montzka, H. Moradkhani, L. Weihermüller, H.-J. H. Franssen, M. Canty, and H. Vereecken, "Hydraulic parameter estimation by remotely-sensed top soil moisture observations with the particle filter," Journal of Hydrology, vol. 399, pp. 410-421, Mar. 2011.

[18] C. Montzka, J. P. Grant, H. Moradkhani, H.-J. H. Franssen, L. Weihermüller, M. Drusch, and H. Vereecken, "Estimation of radiative transfer parameters from 1-band passive microwave brightness temperatures using advanced data assimilation," Vadose Zone Journal, vol. 12, no. 3, 2013. 
[19] J. Qin, S. Liang, K. Yang, I. Kaihotsu, R. Liu, and T. Koike, "Simultaneous estimation of both soil moisture and model parameters using particle filtering method through the assimilation of microwave signal," Journal of Geophysical Research: Atmospheres (1984-2012), vol. 114, no. D15, 2009.

[20] H. Moradkhani, C. M. DeChant, and S. Sorooshian, "Evolution of ensemble data assimilation for uncertainty quantification using the particle filter-markov chain monte carlo method," Water Resources Research, vol. 48, no. $12,2012$.

[21] A. H. Weerts and G. Y. El Serafy, "Particle filtering and ensemble kalman filtering for state updating with hydrological conceptual rainfall-runoff models," Water Resources Research, vol. 42, no. 9, 2006

[22] J. A. Vrugt, C. J. ter Braak, C. G. Diks, and G. Schoups, "Hydrologic data assimilation using particle markov chain monte carlo simulation: Theory, concepts and applications," Advances in Water Resources, vol. 51, pp. 457-478, 2013.

[23] R. Van Der Merwe, A. Doucet, N. De Freitas, and E. Wan, “The unscented particle filter,” in NIPS, pp. 584-590, 2000.

[24] S. Bateni, D. Entekhabi, and D.-S. Jeng, "Variational assimilation of land surface temperature and the estimation of surface energy balance components," Journal of Hydrology, vol. 481, pp. 143-156, 2013.

[25] F. Castelli, D. Entekhabi, and E. Caporali, "Estimation of surface heat flux and an index of soil moisture using adjoint-state surface energy balance," Water Resources Research, vol. 35, no. 10, pp. 3115-3125, 1999.

[26] G. Boni, F. Castelli, and D. Entekhabi, "Sampling strategies and assimilation of ground temperature for the estimation of surface energy balance components," Geoscience and Remote Sensing, IEEE Transactions on, vol. 39, no. 1, pp. 165-172, 2001

[27] S. Margulis, M. Girotto, G. Cortes, and M. Durand, "A particle batch smoother approach to snow water equivalent estimation a particle batch smoother approach to snow water equivalent estimation," Journal of Hydrometeorology, submitted to Journal of Hydrometeorology.

[28] J. Simunek, M. Sejna, H. Saito, M. Sakai, and M. T. Van Genuchten, "The HYDRUS-1D software package for simulating the one-dimensional movement of water, heat, and multiple solutes in variably-saturated media," University of California, Riverside, 2009.

[29] M. T. Van Genuchten, "A closed-form equation for predicting the hydraulic conductivity of unsaturated soils," Soil Science Society of America Journal, vol. 44, no. 5, pp. 892-898, 1980.

[30] M. G. Schaap, F. J. Leij, and M. T. van Genuchten, "Rosetta: A computer program for estimating soil hydraulic parameters with hierarchical pedotransfer functions," Journal of hydrology, vol. 251, no. 3, pp. 163-176, 2001.

[31] S. Lu, T. Ren, Y. Gong, and R. Horton, "An improved model for predicting soil thermal conductivity from water content at room temperature," Soil Science Society of America Journal, vol. 71, no. 1, pp. 8-14, 2007.

[32] H. Saito, J. ŠimÅnek, and B. P. Mohanty, "Numerical Analysis of Coupled Water, Vapor, and Heat Transport in the Vadose Zone," Vadose Zone Journal, vol. 5, no. 2, p. 784, 2006.

[33] A. Doucet and A. M. Johansen, "A tutorial on particle filtering and smoothing: Fifteen years later," Handbook of Nonlinear Filtering, vol. 12, pp. 656-704, 2009.

[34] M. S. Arulampalam, S. Maskell, N. Gordon, and T. Clapp, "A tutorial on particle filters for online nonlinear/nongaussian bayesian tracking," Signal Processing, IEEE Transactions on, vol. 50, no. 2, pp. 174-188, 2002.

[35] J. Judge, J. Casanova, T.-y. Lin, K. Tien, M.-y. Jang, O. Lanni, and L. Miller, "Field observations during the second microwave, water, and energy balance experiment (microwex-2): from march 17 through june 3, 2004," tech. rep., Circular, 2005

[36] S. Eslamian, Handbook of Engineering Hydrology: Modeling, Climate Change, and Variability. Handbook of Engineering Hydrology, CRC Press, 2015.

[37] S. Madadgar and H. Moradkhani, "Improved bayesian multi-modeling: Integration of copulas and bayesian model averaging," Civil and Environmental Engineering Faculty Publications and Presentations, no. 174, 2014.

[38] M. Thyer, B. Renard, D. Kavetski, G. Kuczera, S. W. Franks, and S. Srikanthan, "Critical evaluation of parameter consistency and predictive uncertainty in hydrological modeling: A case study using bayesian total error analysis," Water Resources Research, vol. 45, no. 12, 2009.

[39] S. Dunne and D. Entekhabi, "An ensemble-based reanalysis approach to land data assimilation," Water resources research, vol. 41, no. 2, 2005.

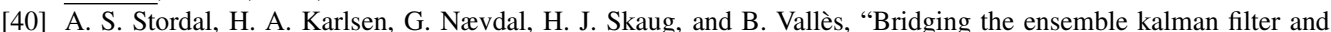
particle filters: the adaptive gaussian mixture filter," Computational Geosciences, vol. 15, no. 2, pp. 293-305, 2011.

[41] H. Moradkhani, S. Sorooshian, H. V. Gupta, and P. R. Houser, "Dual stateparameter estimation of hydrological models using ensemble Kalman filter," Advances in Water Resources, vol. 28, no. 2, pp. 135-147, 2005. 
where $p\left(\mathbf{y}_{t-L+1: t} \mid \mathbf{y}_{1: t-L}\right)$ is a normalize constant [22]. As a result, Equation (A.4) can be simplified as:

$$
p\left(\mathbf{x}_{1: t}^{i} \mid \mathbf{y}_{1: t}\right) \propto p\left(\mathbf{x}_{1: t-L}^{i} \mid \mathbf{y}_{1: t-L}\right) p\left(\mathbf{x}_{t-L+1: t}^{i} \mid \mathbf{x}_{1: t-L}^{i}\right) p\left(\mathbf{y}_{t-L+1: t} \mid \mathbf{x}_{t-L+1: t}^{i}\right)
$$

In the sequential importance sampling algorithm, the particles were sampled from a known distribution (proposal distribution, $\left.q\left(\mathbf{x}_{1: t} \mid \mathbf{y}_{1: t}\right)\right)$ and assigned the weights according to:

$$
w_{t}^{i, *}=\frac{p\left(\mathbf{x}_{1: t}^{i} \mid \mathbf{y}_{1: t}\right)}{q\left(\mathbf{x}_{1: t}^{i} \mid \mathbf{y}_{1: t}\right)}
$$

s46 substituting $p\left(\mathbf{x}_{1: t}^{i} \mid \mathbf{y}_{1: t}\right)$ with Equation (A.5), it gives:

$$
w_{t}^{i, *} \propto \frac{p\left(\mathbf{x}_{1: t-L}^{i} \mid \mathbf{y}_{1: t-L}\right) p\left(\mathbf{x}_{t-L+1: t}^{i} \mid \mathbf{x}_{1: t-L}^{i}\right) p\left(\mathbf{y}_{t-L+1: t} \mid \mathbf{x}_{t-L+1: t}^{i}\right)}{q\left(\mathbf{x}_{1: t}^{i} \mid \mathbf{y}_{1: t}\right)}
$$

547 Similar with the PF algorithm [23], a proposal distribution of the following form is used:

$$
q\left(\mathbf{x}_{1: t}^{i} \mid \mathbf{y}_{1: t}\right)=q\left(\mathbf{x}_{t-L+1: t}^{i} \mid \mathbf{x}_{1: t-L}^{i}, \mathbf{y}_{1: t-L}\right) q\left(\mathbf{x}_{1: t-L}^{i} \mid \mathbf{y}_{1: t-L}\right)
$$

Combining Equation (A.7) and (A.8), the weights can be arranged as:

$$
\begin{aligned}
w_{t}^{i, *} & \propto \frac{p\left(\mathbf{x}_{1: t-L}^{i} \mid \mathbf{y}_{1: t-L}\right)}{q\left(\mathbf{x}_{1: t-L}^{i} \mid \mathbf{y}_{1: t-L}\right)} \frac{p\left(\mathbf{x}_{t-L+1: t}^{i} \mid \mathbf{x}_{1: t-L}^{i}\right) p\left(\mathbf{y}_{t-L+1: t} \mid \mathbf{x}_{t-L+1: t}^{i}\right)}{q\left(\mathbf{x}_{t-L+1: t}^{i} \mid \mathbf{x}_{1: t-L}^{i}, \mathbf{y}_{1: t-L}\right)} \\
& \propto w_{t-L}^{i, *} \frac{p\left(\mathbf{x}_{t-L+1: t}^{i} \mid \mathbf{x}_{1: t-L}^{i}\right) p\left(\mathbf{y}_{t-L+1: t} \mid \mathbf{x}_{t-L+1: t}^{i}\right)}{\left.q\left(\mathbf{x}_{t-L+1: t}^{i}\right) \mid \mathbf{x}_{1: t-L}^{i}, \mathbf{y}_{1: t-L}\right)}
\end{aligned}
$$


${ }_{548}$ Similar to the PF, this study uses the distribution of $p\left(\mathbf{x}_{t-L+1: t}^{i} \mid \mathbf{x}_{1: t-L}^{i}\right)$ for $q\left(\mathbf{x}_{t-L+1: t}^{i} \mid \mathbf{x}_{1: t-L}^{i}, \mathbf{y}_{1: t-L}\right)$.

${ }_{549}$ Consequently, Equation (A.9) is reduced to:

$$
w_{t}^{i, *} \propto w_{t-L}^{i, *} p\left(\mathbf{y}_{t-L+1: t} \mid \mathbf{x}_{t-L+1: t}^{i}\right)
$$

550 The normalized weight is given by:

$$
w_{t}^{i}=\frac{w_{t}^{i *}}{\sum_{i=1}^{N} w_{t}^{i *}}
$$

551 $552 w_{t}^{i}$.

For the PBS algorithm, the calculated weight is applied for the entire window, i.e. $w_{t-L+1: t}^{i}=$ 
TABLES

Table .1: Generation of perturbed inputs (soil property and forcing) for each particle member.

\begin{tabular}{l|llll}
\hline Variable & Error Distribution & Mean & Standard deviation & Bound \\
\hline Sand $(\%)$ & Uniform & - & - & 55,95 \\
Silt $(\%)$ & Uniform & - & - & 0,20 \\
$\rho_{b}\left(\mathrm{~g} / \mathrm{cm}^{3}\right)$ & Gaussian, Additive & $0\left(\mathrm{~g} / \mathrm{cm}^{3}\right)$ & $0.05\left(\mathrm{~g} / \mathrm{cm}^{3}\right)$ &,-- \\
Precipitation $(\mathrm{mm})$ & Gaussian, Multiplicative & 1 & $\times 0.2$ &,-- \\
Radiation $\left(\mathrm{W} / \mathrm{m}^{2}\right)$ & Gaussian, Multiplicative & 1 & $\times 0.075$ &,- 1350 \\
Air temperature $\left({ }^{\circ} \mathrm{C}\right)$ & Gaussian, Multiplicative & 1 & $\times 0.05$ &,-- \\
Relative humidity $(\%)$ & Gaussian, Multiplicative & 1 & $\times 0.05$ &,- 100 \\
Wind speed $(\mathrm{Km} / \mathrm{h})$ & Gaussian, Multiplicative & 1 & $\times 0.2$ &,-- \\
\hline
\end{tabular}

Table .2: Comparison of soil moisture RMSE $\left(m^{3} m^{-3}\right)$, Bias $\left(m^{3} m^{-3}\right)$ and Reliability estimated using the PF and PBS at five layers. The value presented are the averaged RMSE from 20 tests. The observation interval being considered are 15 minutes (PF-15min and PBS-15min) and 45 minutes (PF-45min and PBS-45min). The window length for the PBS is 3 hours.

\begin{tabular}{lllllll} 
Meurs. & \multirow{5}{*}{ Metric } & Algorithm & \multicolumn{5}{c}{ Depths } \\
\cline { 3 - 6 } & & $\mathbf{2 c m}$ & 4cm & $\mathbf{8 c m}$ & $\mathbf{3 2 c m}$ & $\mathbf{6 4 c m}$ \\
\hline RMSE & PF-45min & 0.037 & 0.039 & 0.045 & 0.062 & 0.094 \\
& PBS-45min & 0.035 & 0.037 & 0.042 & 0.059 & 0.094 \\
& PBS-15min & 0.029 & 0.029 & 0.030 & 0.039 & 0.074 \\
\hline Bias & PF-45min & 0.029 & 0.035 & 0.042 & 0.059 & 0.098 \\
& PBS-45min & 0.027 & 0.032 & 0.036 & 0.052 & 0.090 \\
& PBS-15min & 0.019 & 0.020 & 0.023 & 0.033 & 0.081 \\
\hline Reliability & PF-45min & 0.087 & 0.031 & 0.021 & 0.002 & 0.000 \\
& PBS-45min & 0.143 & 0.074 & 0.053 & 0.004 & 0.000 \\
& PBS-15min & 0.346 & 0.314 & 0.155 & 0.004 & 0.000 \\
\hline
\end{tabular}




\section{FIGURES}

\section{The updating procedure in the Particle Filter}

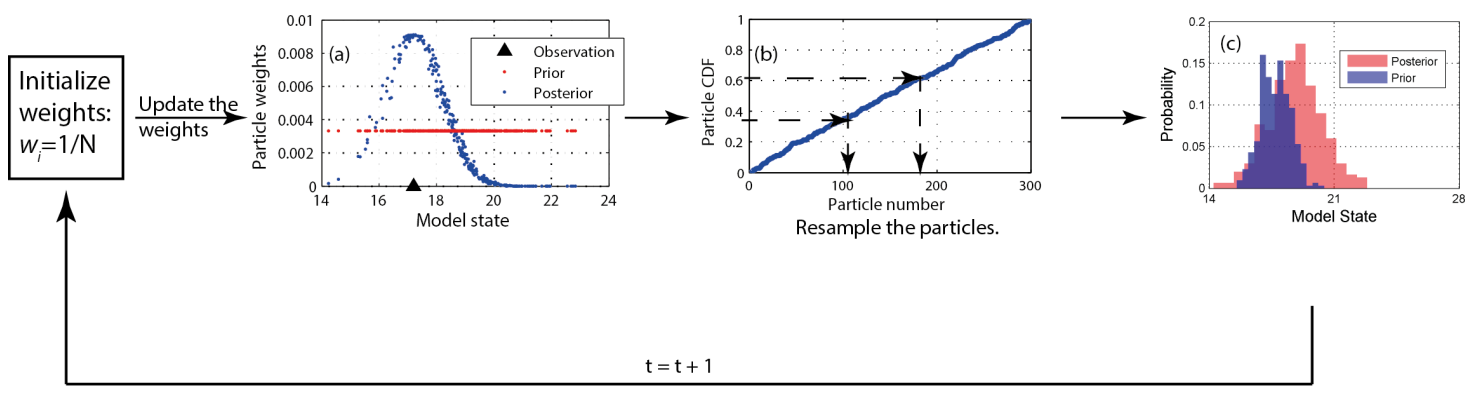

The moving window strategy in the Particle Batch Smoother
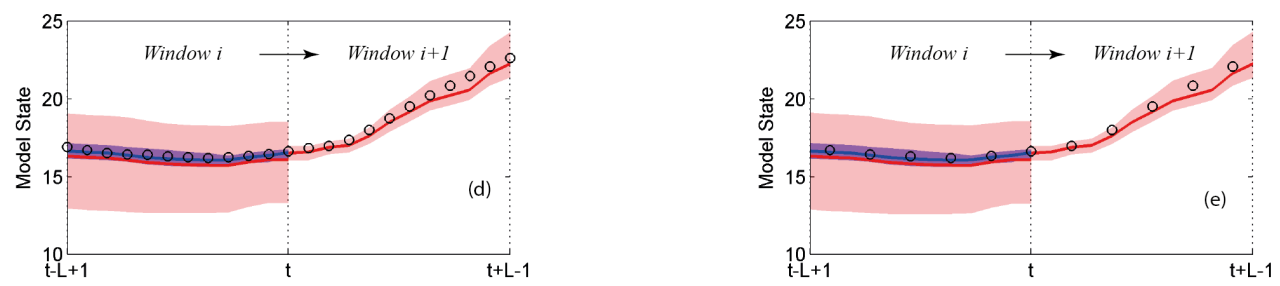

Figure .1: A diagram for illustrating the Particle Filter (PF) at one updating step (upper panel) and the Particle Batch Smoother (PBS) moving window strategy (lower panel). In the PBS moving window strategy plot, the solid lines are the mean of the prior and posterior, and the distributions of the prior and posterior are shown as the shaded red and blue area. Black circles represent the observations, and the strategy of using different observation interval in the PBS is shown in (d) and (e) respectively 

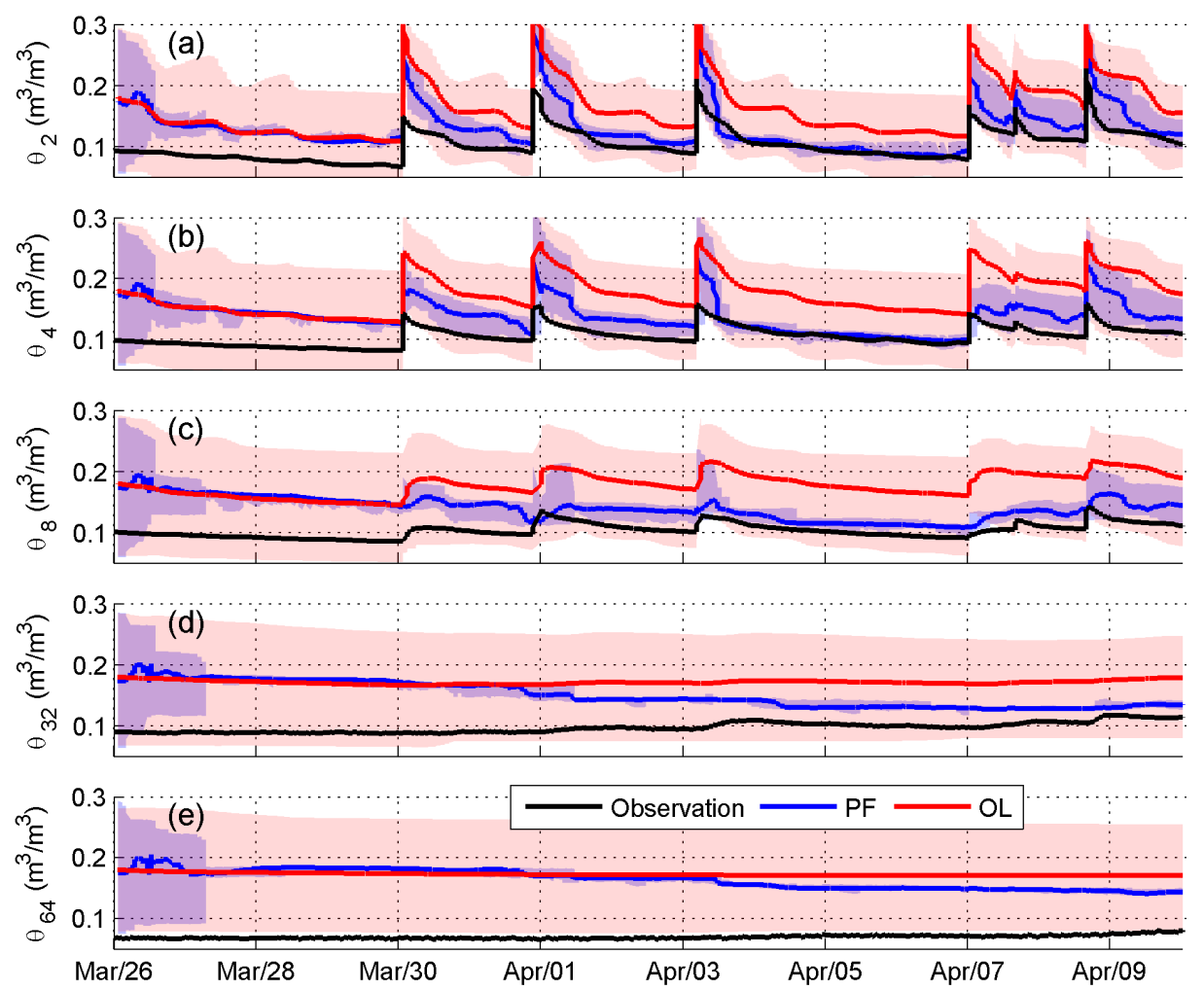

Figure .2: Comparison of soil moisture estimated by the open loop (OL) and the particle filter (PF) with observations at 5 depths. The PF algorithm in this case updates soil moisture using temperature observations every $45 \mathrm{~min}$. The Solid lines for the OL and the PF are the mean of the particles. The shaded area are the range of the particles for the OL (red) and the PF (blue). 
(a) Temperature at $4 \mathrm{~cm}$

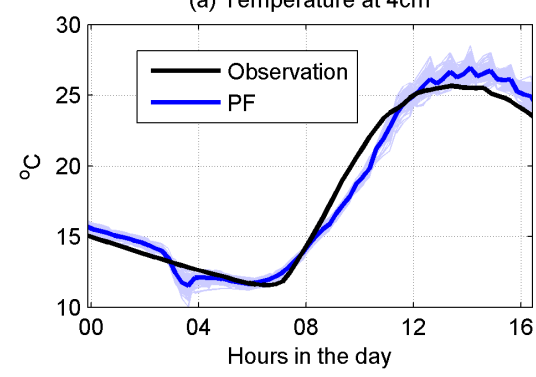

(c) Moisture at $4 \mathrm{~cm}$

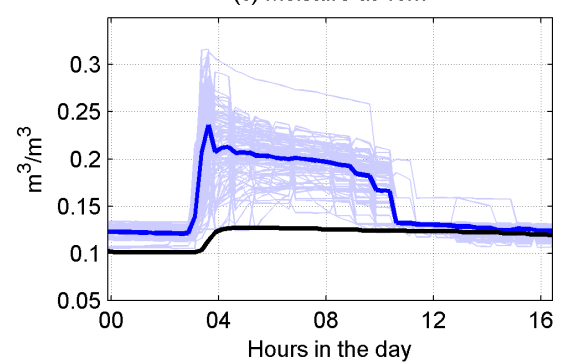

(b) Temperature at $8 \mathrm{~cm}$

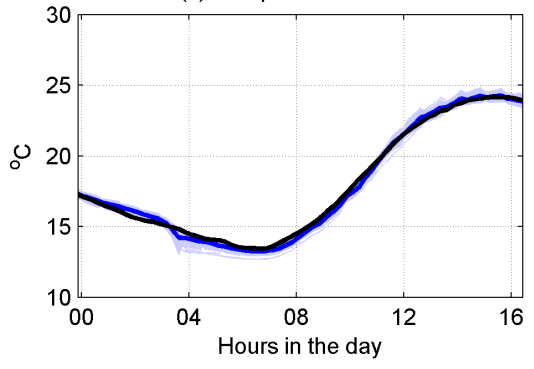

(d) Moisture at $8 \mathrm{~cm}$

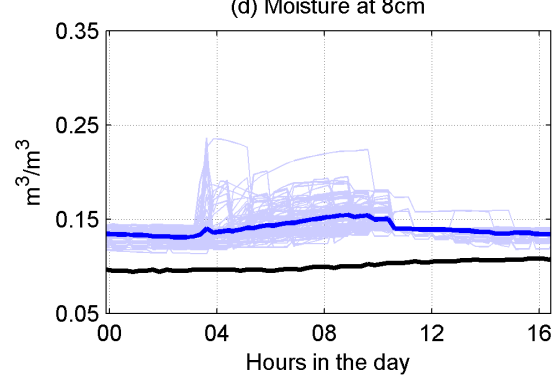

Figure .3: Soil temperature and moisture estimates at 4 and $8 \mathrm{~cm}$ on 2 nd April. The thin blue lines represent the ensemble members.

(a) $\theta_{2 \mathrm{~cm}}$

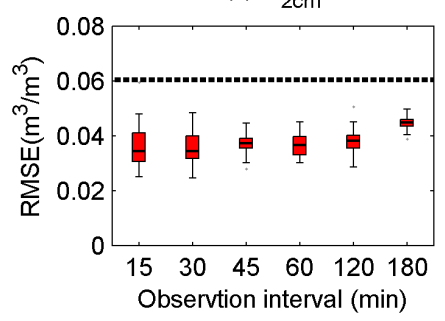

(d) $\theta_{32 \mathrm{~cm}}$

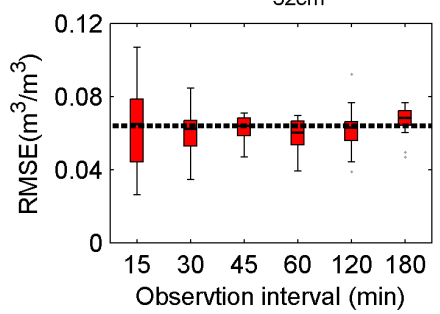

(b) $\theta_{4 \mathrm{~cm}}$

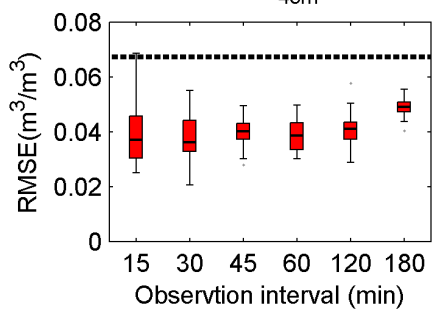

(e) $\theta_{64 \mathrm{~cm}}$

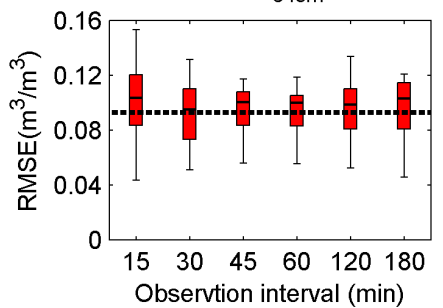

(c) $\theta 8 \mathrm{~cm}$

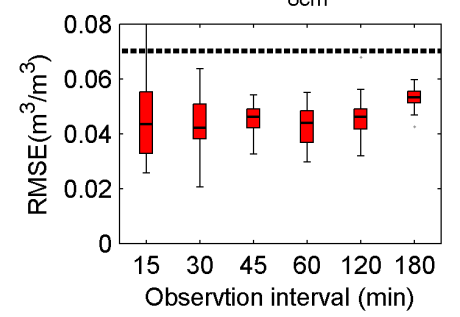

Figure .4: Comparison of RMSE of soil moisture estimated using the PF with different data observation intervals. The PF was tested using different initialized model inputs, and 20 runs were used for each observation interval. The black dashed line represents the RMSE of the open loop. In the box plot, the middle black line denotes the median value, the edges of the box are the interquartile range (IQR), the maximum length of the whiskers is set to be the 1.5 times of the IQR, and values larger/smaller than the maximum/minimum the whiskers are considered as outliers (black dots). The legends are the same for the following boxplots. 
(a) $\theta_{2 \mathrm{~cm}}$

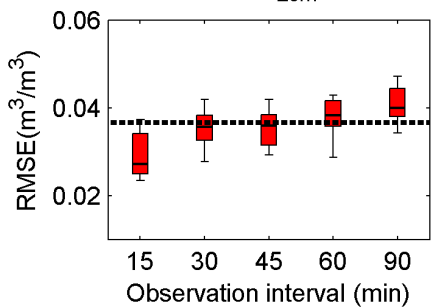

(d) $\theta_{32 \mathrm{~cm}}$

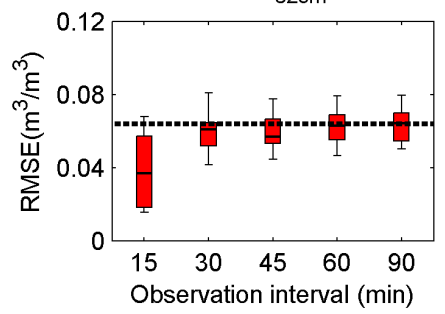

(b) $\theta_{4 \mathrm{~cm}}$

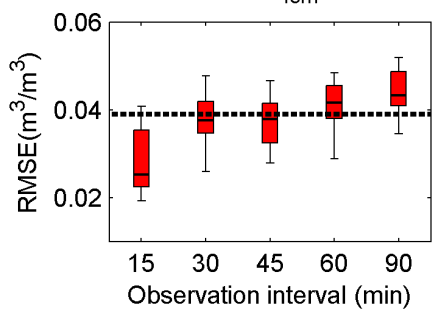

(e) $\theta_{64 \mathrm{~cm}}$

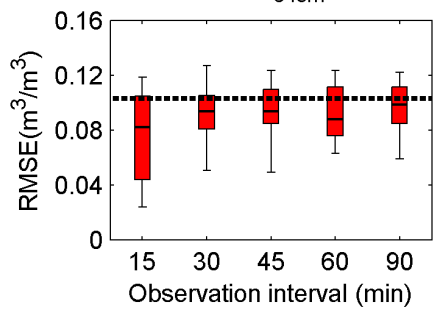

(c) $\theta_{8 \mathrm{~cm}}$

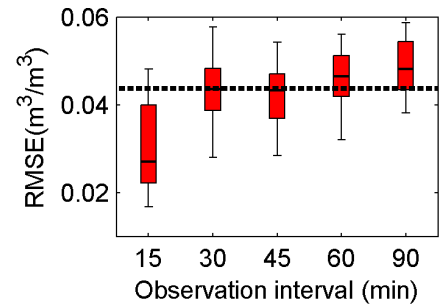

Observation interval (min)

Figure .5: Impacts of soil temperature observation interval on the PBS algorithm. The PBS algorithm uses a 3 hour window length with 100 particles. The black dashed line represents the averaged RMSE of the estimates from the PF using an observation interval of 45 minutes. Similar to Fig .4, 20 tests with different model initialization are used.

(a) $\theta_{2 \mathrm{~cm}}$

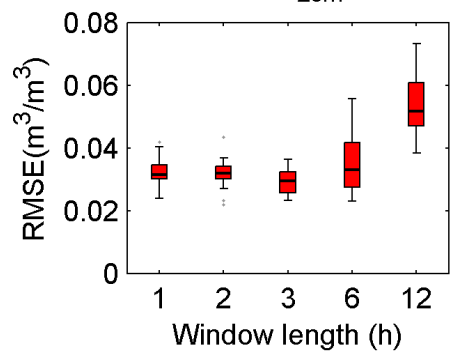

(d) $\theta_{32 \mathrm{~cm}}$

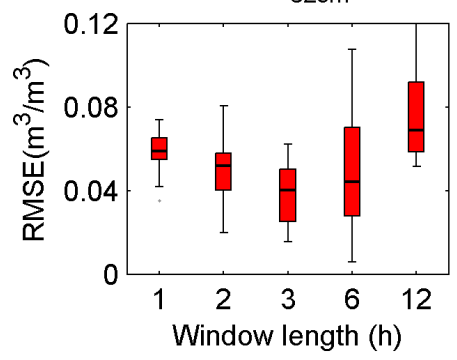

(b) $\theta_{4 \mathrm{~cm}}$

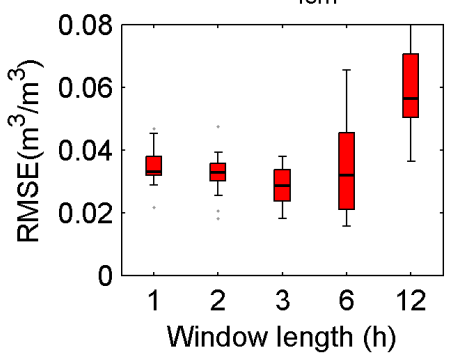

(e) ${ }^{6} 64 \mathrm{~cm}$

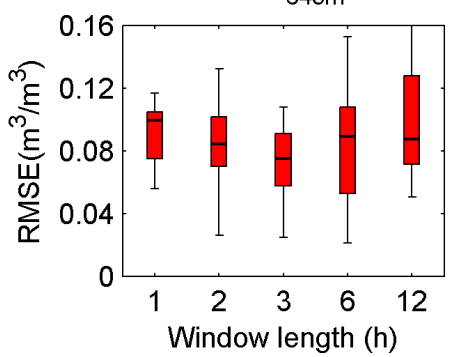

(c) $\theta$ $8 \mathrm{~cm}$

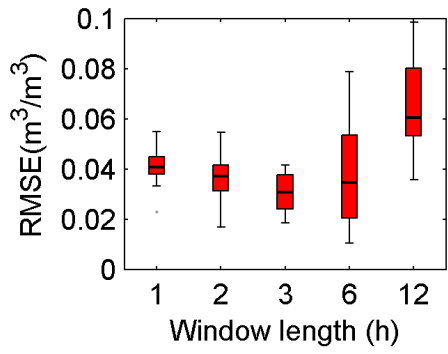

Window length $(\mathrm{h})$

Figure .6: Comparison of RMSE of soil moisture estimated using the PBS with different assimilation window lengths at 5 depths. For each window length, 20 tests with different model initialization is used. The observation interval is 15 minutes, with 100 particles. 

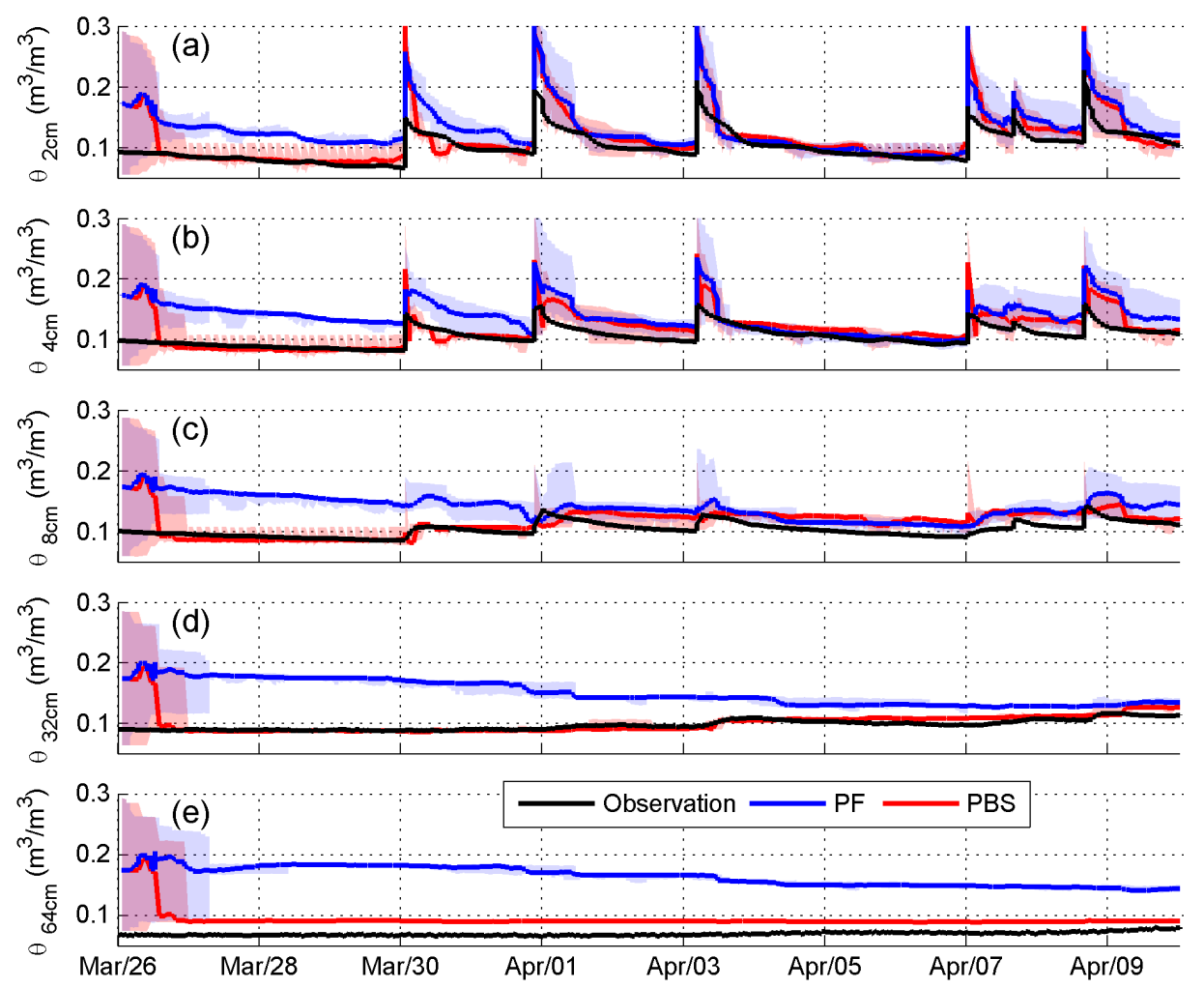

Figure .7: An illustrative example of comparing the soil moisture estimates at 5 depths using the optimal PF (observation interval of 45 minutes), and the the optimal PBS (observation interval of 15 minutes and window length of 3 hours). The blue and red solid lines represent the mean of the PF and the PBS estimates. The shade areas are the particle range of the PF (blue) and the PBS (red). The initial conditions for the PBS and PF are the same, and identical to these used in Fig.2. 

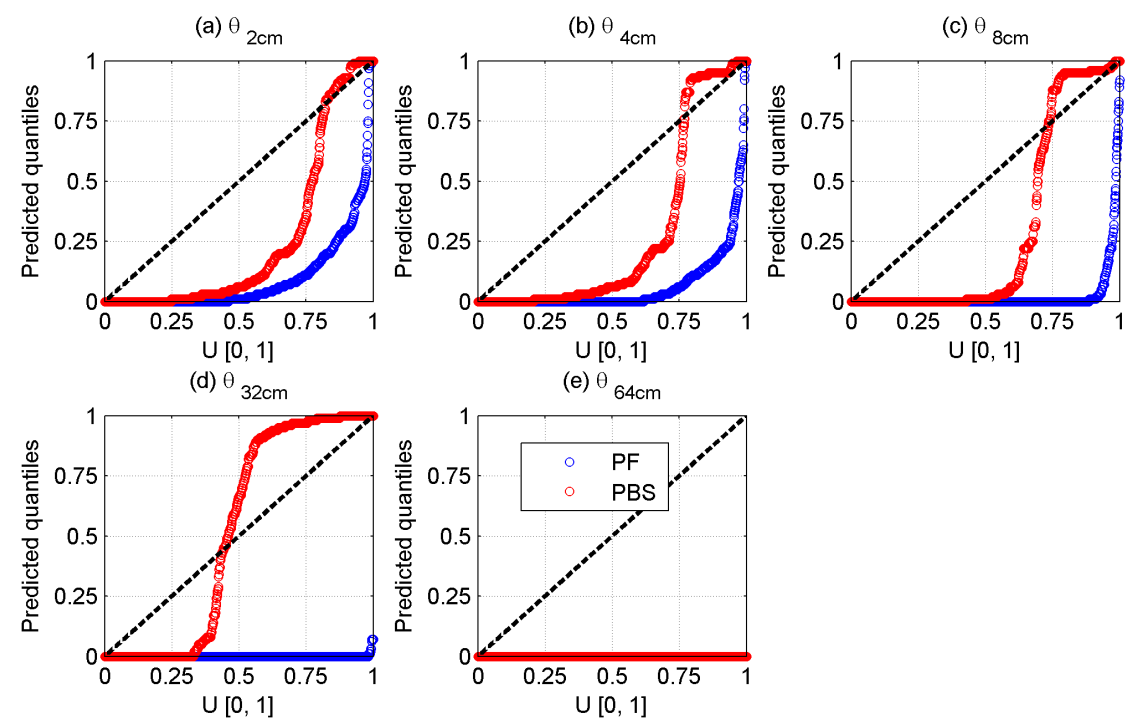

Figure .8: The QQ plot for the PF and the PBS estimated soil moisture at five depths. The estimates from the results presented in Fig.7. 

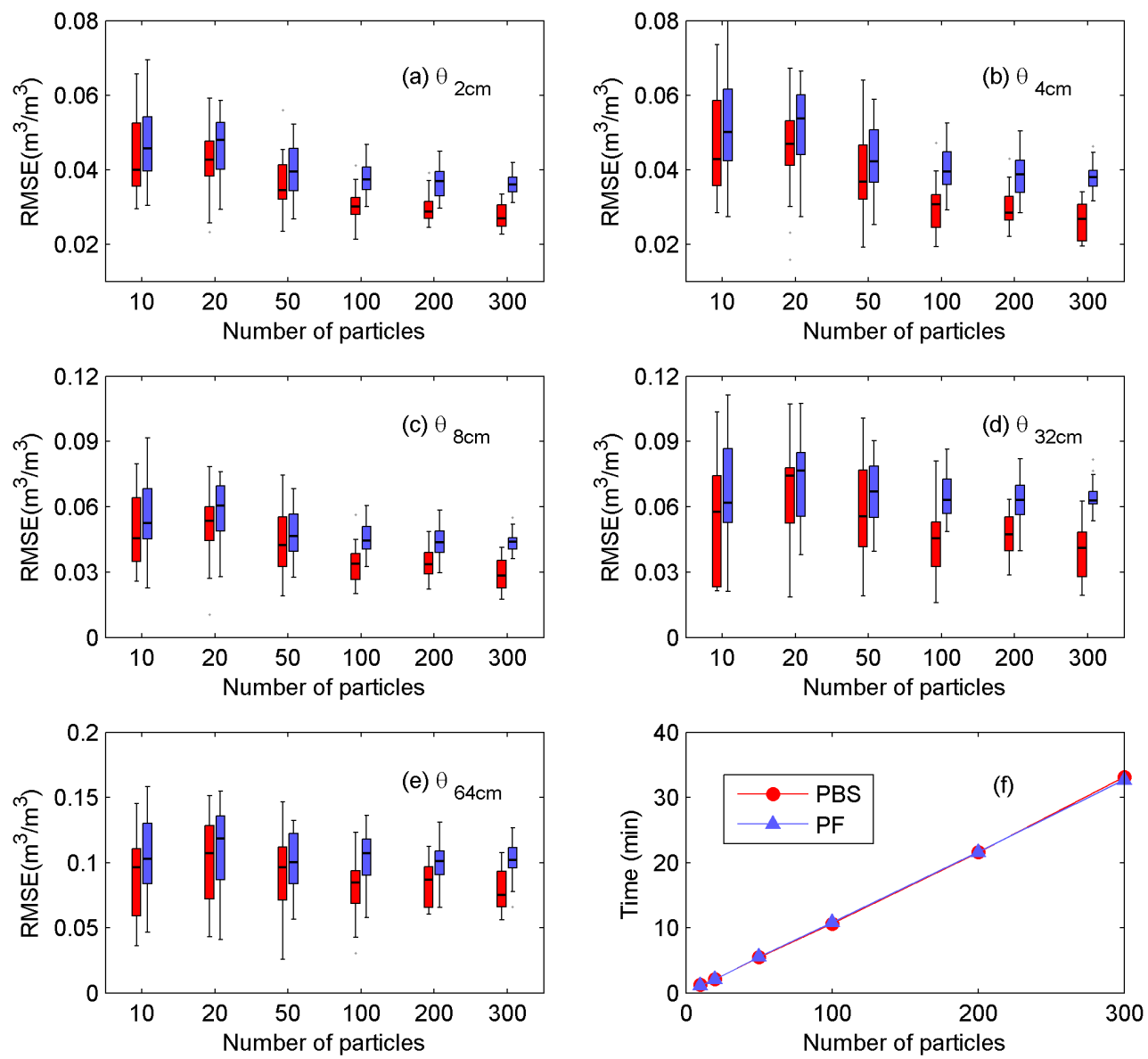

Figure 9: Comparison of RMSE of soil moisture estimated using the PBS and the PF with different number of particle sizes at 5 depths (a to e), and the computation time for different number of particles (f). A window length of 3 hours and 15 minutes observation interval is used for the PBS. The observation interval for the PF is 45 mintues. For each window length, 20 different initialized model inputs were used for testing. 